\title{
A Hierarchical Adaptation Framework for Adaptive Training Systems
}

\author{
Sven Fuchs, Angela Carpenter, Meredith Carroll, and Kelly Hale \\ Design Interactive, Inc. 1221 E Broadway, \\ Oviedo, FL 32765, USA \\ \{sven, angela, meredith, kelly\} @designinteractive.net
}

\begin{abstract}
Real-time adaptation is challenging in both operational and training environments, as the system must be able to identify what, why, and when mitigation is needed, and how best to mitigate to optimize the human-system interaction. Training systems have additional complexities, as the sole goal is not to optimize performance as in operational environments, but to optimize training, which may involve more error allowance for learning opportunities. This paper outlines a proposed hierarchical adaptation framework for adaptive training systems, involving diagnoses of learning state, performance, and expertise. It will also discuss candidate approaches to obtaining the necessary measurements using physiological and neurophysiological processes, provide some guidance for designing strategies for optimal adaptation, and highlight current challenges and future research areas.
\end{abstract}

Keywords: Adaptive Training, Augmented Cognition, Training Systems.

\section{Introduction}

Adaptive training has great potential across many domains, including airport baggage screening, which involves human review of electronic images/video of bags in search of explosives and other dangerous items. Screening is a repetitive visual search task that often has a very low probability of encountering a threat, but extremely high consequences if a serious threat is missed. Due to the importance of screening accuracy, screeners are required to complete extensive training both before going on the job and while employed. The unrepresentative ratio of threat targets during training (to ensure exposure within constrained timeframe) and repetitiveness of current training practices over time degrades its effectiveness, and current evaluation methods are unable to quantitatively measure details of officer state such as hazardous cognitive states (e.g., fatigue, boredom, inattention) that could negatively impact training outcomes and identify root cause(s) of performance errors (i.e., scan vs. recognition error) and influencing factors (e.g., threat type, location, orientation, clutter in bag, etc.). Instead, instructors using traditional training methods are often limited to observable behavioral metrics (e.g., detections, false alarms).

To more completely identify all obstacles to training effectiveness in this domain and others, new training systems are required that 'peer into the mind of the users' to capture perceptual and cognitive processes not otherwise accessible, and provide 
quantitative metrics to evaluate trainee state throughout a training session. This would result in a more complete measurement suite that allows a comprehensive diagnosis process to identify root cause(s) of training deficiencies/inefficiencies that can in turn provide directed feedback for addressing issues, thus individualizing training to optimize training effectiveness and efficiency. Such a training system could provide in-action feedback, where training scenarios can adapt in real-time to provide individualized training targeted at specific error patterns. Furthermore, such a training system could provide a comprehensive After Action Review (AAR) display that summarizes training strengths, deficits, and provides playback options (e.g., freeze in action, step through action) to highlight training opportunities. A thorough understanding of when to adapt, what to adapt, and how to adapt to optimize training is required to ensure that deficiencies/inefficiencies are targeted at appropriate times to focus training and avoid negative adaptation consequences such as distraction or confusion. There is a need to develop tools that support instructors and screeners to address when errors occurred, what errors occurred and why these errors occurred to increase the diagnostic value of training assessment.

Currently, no operational training systems incorporate this level of advanced performance measurement. While many testbeds have been developed to study these tools in a laboratory setting, few have addressed the many theoretical and technical challenges associated with developing a fieldable system. First, the sensor hardware must be usable and noninvasive while maintaining high levels of accuracy and reliability. Second, the system must facilitate near real time correlation of sensor data with scenario and behavioral events. Third, the system must facilitate the processing of very granular process level data into meaningful actionable diagnoses. Fourth, the system must respond to these diagnoses in a manner that ideally addresses the training gaps and learning state of the trainee.

This paper will outline a proposed adaptation framework for diagnoses of learning state, performance, and expertise, discuss candidate approaches to obtaining the necessary measurements using physiological and neurophysiological processes, and provide some guidance for designing strategies for optimal adaptation. Current challenges and future research areas are also discussed.

\section{A Training Adaptation Framework}

Adaptive training systems are different from their operational counterparts in that performance optimization is not the only goal of the adaptation. In a training setting, the objective is to train efficiently and effectively, which often requires the user to make mistakes as an acceptable and valuable part of learning. Such "mistake" opportunities may be lost (mitigated or prevented) in a performance-optimizing operational system. While adaptations are used operationally to mitigate problem states (a "treat the symptom" approach), adaptations should be used in training to address root causes of problem states, as training allows time and flexibility to drill down to that level. Conversely, while good performance is the goal of operational systems, it may signal the need for adaptation in a training environment if the trainee has mastered a skill and is ready to move on. 
In order to effectively adapt a scenario without disrupting learning, use of a hierarchical adaptation framework is proposed. At the highest priority level (Level 1), it should be ensured that the situation affords learning and that learning can occur. Given this, it is first necessary to mitigate negative learning states such as drowsiness and distraction. Given the example vigilance task, these states are anticipated to occur and could conceal true performance issues and prevent training opportunities. The second priority (Level 2) is to address skill deficiencies, guiding trainees to acquire all necessary knowledge and skills, preventing them from practicing bad habits, and perpetuating incorrect performance or error patterns. Once performance is adequate, the third priority (Level 3) is to target development of expertise, providing trainees with practice opportunities and instruction designed to move them up the expertise continuum to automated performance. Based on these three diagnostic categories (i.e., Learning State, Skill, and Expertise), it is necessary to identify how data would be obtained and integrated to diagnose adaptation trigger points. Candidate approaches are discussed in the following section.

\section{Measurement and Diagnosis}

Given the 'unobservable' perceptual and cognitive processes involved in many complex tasks, it may be necessary not only to assess outcomes such as accuracy of response, but also to assess process measures at a cognitive level. To realize such an adaptive training solution, advances in both measurement/diagnosis and in training science could be utilized that address the framework's information requirements and enable effective and efficient adaptation to optimize training. A comprehensive suite of metrics and diagnostic capabilities is needed to drive such individualized feedback to optimize training effectiveness and efficiency. Advances made in psychophysiological sensing technology have demonstrated success in evaluating cognitive state in near real-time. Numerous sensors exist to capture process measures of cognitive activity, including behavioral, physiological and brain-based technology. These technologies provide insights that go beyond outcome performance and beyond the capabilities of an observing instructor.

According to the proposed training adaptation framework, adaptations to training should be made with the goals of first enhancing readiness for learning to ensure the information provided during training can be effectively processed by the trainee, second, improving knowledge and skills to allow development of skilled performance, and third, increasing expertise levels to boost efficiency and effectiveness of both training and trainee performance. In the following subsections, the three diagnostic approaches - learning state diagnosis, skill diagnosis, and expertise diagnosis - will now be discussed in detail.

\subsection{Learning State (Readiness) Diagnosis}

With the emergence of neurophysiological and physiological measurement technology that allows for real-time assessment of perceptual and cognitive processing, many unobservable processes become accessible. Specifically, some cognitive states that are 
measurable via electroencephalography (EEG), including workload and engagement, can provide neurophysiological measures of the unobservable aspects of cognition [1, 2]. The following list outlines specific cognitive states that generally negatively affect the readiness for training by reducing attentional resources that facilitate learning and retention. Thus, it may be possible to utilize certain neurophysiological cognitive state metrics to detect issues with readiness to learn:

- Workload: High cognitive workload is expected when performing in a knowledgebased control mode because no automaticity guides the process [3, 4]. In rulebased control mode, rules are consciously retrieved from memory and applied to gathered information, also causing increased cognitive processing demands. Experts using skill-based techniques, however, employ automated routines that require fewer cognitive resources. Thus, it is expected that the assessment of cognitive workload can contribute to the identification of the trainee's control mode.

- Engagement: Because of high task demands, novice and journeyman trainees are expected to exhibit higher levels of engagement than expert trainees because studies have shown a trend for decreasing EEG engagement with increasing task proficiency $[3,5]$.

- Distraction: Distraction is a state characterized by a lack of clear and orderly thought and behavior, where a trainee becomes involved somewhere other than the cognitive tasks of interest [6]. Expert performers have an exhaustive mental model of the task or situation so that very few situations cause distraction. Confusion is one element of distraction. In rule-based decision makers, confusion may stem from the conscious selection of rules and difficulties in applying them to the situation at hand. Naïve trainees are expected to show relatively high levels of confusion because their mental models are more likely to be incorrect or insufficient so that new situations may cause a mismatch.

- Drowsiness: Sleep disorders are common and can have deleterious effects on performance $[7,8,9]$. In fact, loss of sleep can accumulate over time and result in a "sleep debt," which can lead to impairments in alertness, memory, and decision making. Individuals with chronic accumulation of fatigue are often unaware of the impact on their performance.

\subsection{Skill Diagnosis}

Skill development is typically measured using performance metrics that are built into the training environment. Typical examples are "time to engage" or "number of hits". However, these metrics do not provide information about why performance breakdowns occur, so that traditional training often addresses symptoms by offering repeat practice instead of addressing the root cause(s) of errors and/or error patterns. Using the baggage screener example, it would not be possible to provide proper feedback without knowing whether a threat was missed due to improper visual scanning patterns (i.e., the trainee did not look at the threat or did not dwell on the threat long enough to allow cognitive processing of threat) or recognition issues (i.e., screener visually interrogated threat, but did not correctly identify it as such). 
For training involving perceptual tasks (which is the case for most training environments), the addition of eye tracking can add a level of richness in evaluation that cannot be accomplished by only monitoring overt behavioral responses. Processes that can be detected by eye tracking but are otherwise hard to capture are those associated with perceptual-cognitive evaluation (i.e., perceptual-cognitive processes that do not necessarily have an immediate observable response). Instead, the first response to a changed situation is often information gathering, evaluation and decision making, all of which are non-behavioral activities. The addition of eye tracking enables the collection of real-time quantitative and qualitative data indicative of the perceptual-cognitive process between a stimulus and the (behavioral) response.

Eye tracking also aids in the assessment of perception through measurement of visual attention via gaze, scan path, and fixation data. The metrics can identify whether and what Areas of Interest (AOIs) were gazed upon, how often, and the duration of the visual attention. By examining attention shifts during an observation task (e.g., to relevant AOIs after receiving a command), eye tracking data can provide contextual information (i.e., what is or is not being attended to) to enhance performance diagnosis. Additionally, eye tracking performance criteria can be developed to determine if a trainee is applying effective scanning strategies, looking at the correct cues, and if they are completing the perceptual tasks efficiently.

Physiological metrics could also be combined with other measures to provide a more holistic picture of performance. Continuous monitoring of cognitive state and performance metrics can provide indications of task vigilance without altering the task flow, especially during low-frequency target detection tasks like baggage screening. Visual scan and focal patterns could be identified to indicate whether trainees (1) scanned appropriate areas, (2) dwelled on potential threats, and (3) combined with behavioral outcomes, determine whether appropriate threats were correctly identified. Results of this analysis can be used to drive discrete training interventions that address scan vs. detect vs. recognition errors. One could also develop an individualized neural profile of brain states coincident with low and high performance periods to obtain insights into perceptual and cognitive processing. Assessing underlying skills associated with identifying relevant information versus distractors could drive error pattern understanding to identify key parameters that should be the focus of future training.

\subsection{Expertise Diagnosis}

To develop expertise, it is necessary to train beyond simple skill acquisition. Practicing skills from different angles and acquiring strategies for more efficient processing is necessary to reach a level of proficiency at which the processes related to each skill have become easily accessible and can be executed in an automated manner. Expertise diagnostics could also replace the instructor's ability to aggregate all available information to avoid burdening the user with irrelevant information or inappropriate training pace or complexity.

It is proposed that physiological indicators can be used in conjunction with behavioral metrics and performance indicators to identify levels of expertise. Prior research [10] has identified a domain general control network that supports cognitive tasks involved in the learning of new tasks and is comprised of lateral frontal (goal 
processing and task switching), medial frontal (process monitoring, decision making, conflict management), and poster parietal (attentional control) brain regions. In general, these areas "drop-out" after extended training. This has been described [11] as a "processing efficiency change" that predicts increased cortical idling (alpha activity) with the development of expertise. As a trainee transitions from novice to advanced learner in a given task domain, cortical support transitions from the control network to task-specific processing regions that are more efficient and characterized as automatic processing. Accordingly, EEG activity reflects this change with increased alpha activity in the control network and more focal beta and gamma activity (slight decrease compared to novices due to processing efficiency) in task-specific regions. This is consistent with the neural efficiency hypothesis of expertise since experts deliver greater performance with seemingly less high frequency cortical activation. The widespread increases in alpha activity essentially "quiets" the brain which increases the salience of the focal higher frequency activation, even though it is relatively low levels, which supports expert task performance. Based on these findings, the hypothesis for driving expertise evaluation is that low frequency control network activity will dramatically increase with expertise whereas high frequency task-specific activity will slightly decrease.

Identification of expertise levels has important implications for implementing an adaptive training solution that considers trainee progress (i.e., transformation), and actively and effectively transitions trainees from one expertise level to the next one until proficiency is reached. Experts are said to learn more from their mistakes than from correct responses [12], and thus "tough cases" that are meant to instigate errors will be used in order for trainees to learn how to handle complexity, overcome knowledge shields (i.e., rationalization of misunderstandings), and guide them in acquiring more adaptive knowledge that can be used to address the unexpected (i.e., tough cases) [13]. Once trainees have reached a high level of competency for a particular set of procedural skills, training can be automatically refocused on other skill areas to ensure optimal use of training resources. At this level refresher training may also occur to maintain proficiency.

\section{Adaptation Strategies}

Once mechanisms are in place to support the collection of data and calculation of diagnostics required for the hierarchy levels, adaptation strategies are necessary to enable the closed-loop adaptive training system to react to these insights. Specifically, adaptation triggers and training strategies should be developed to (1) address nonoptimal learning states (e.g., drowsiness, distraction) that require mitigation, (2) address skill-related deficiencies/inefficiencies which need remediation, and (3) adapt to changes in expertise. The goal should be to develop considerate, context-sensitive adaptations that exhibit minimal cognitive cost.

Generally, two types of adaptation strategies can be distinguished: real-time interventions that are triggered during the course of training, and between-scenario adaptations that are based on more global or composite measures and are typically invoked after a training section or lesson is completed. Real-time mitigations need to seamlessly integrate into the training scenario so as to guide/aid as needed to optimize 
learning and training transfer. Examples are feedback cues, instructional messages, and adaptation of training difficulty. Adaptations occurring between scenarios can be used to alter the training course, training method, or the skill set being trained.

\section{Future Work}

The above framework and approaches constitute an early conceptual approach to accomplishing truly adaptive training that has the potential to revolutionize training effectiveness and efficiency. However, many of the outlined approaches and strategies are still at infancy and advancements in several areas are needed to fully realize the potential of dynamic training adaptation.

One important consideration is the level of individualization. Multiple factors will impact the effectiveness of mitigation strategies implemented, including the training context and scenario content, and individual differences, as some strategies may be more effective for a given individual compared to another trainee. For example, some trainees may be visual learners, and thus respond optimally to a visual representation of corrective action, while others may best learn via spoken instruction/correction. Thus, the ideal mitigation framework would be adaptive itself, and learn over time which strategies work best for a given individual, thereby continuously optimizing the individual training experience.

Other challenges are the technical limitations of the measurement equipment. Physiological sensors must become cheaper and less intrusive to gain broad user acceptance while maintaining or even improving accuracy. Time-synching of multiple data streams, as well as practical issues in diagnosis design such as classifier development, state/skill/expertise threshold establishment, and pattern identification are additional areas with tremendous improvement potential.

Extending to team training settings, adaptation design challenges further increase, as some adaptation strategies (e.g., adapting timing parameters of tasks) may not be effective in a team training environment. For example, slowing down the pace of a scenario designed to assist one trainee in understanding the intricacies of the training may negatively impact team members' performance by slowing down the flow of information and/or changing the order of information flow which may result in negative training transfer. Therefore, the impact of adaptations on team settings must be considered and new team-wide adaptation strategies must be developed.

\section{Conclusions}

Advances in adaptive training are addressing some of the limitations of current performance assessment and training. It has become apparent that a one size fits all philosophy does not make for successful training. Valuable simulation time is lost and training quality is compromised when trying to force all students through the exact same curriculum on the same schedule. Likewise, relying on expert assessment of student performance within a dynamic environment such as a simulation is not always effective or reliable. It is easy for even the expert observer to miss important details of a student's performance in a rapidly unfolding scenario. 
This insight has led to a wide-spread increase of research into adaptive training approaches, and the framework described in this paper is the result of such efforts. Although considerable conceptual and technological challenges exist, the identification of limitations is an important step towards addressing them. Once acknowledged, future efforts can be focused appropriately to address them in a targeted manner, so that the full potential of adaptive training can soon be realized.

Acknowledgments. This material is based upon work supported in part by the Office of Naval Research (ONR) under SBIR contracts N00014-09-M-0385 and FA8650-08M-6826, and the Department of Homeland Security (DHS) under SBIR contract N10PC20028. Any opinions, findings and conclusions or recommendations expressed in this material are those of the authors and do not necessarily reflect the views or the endorsement of ONR and DHS.

\section{References}

1. Dorneich, M.C., Whitlow, S.D., Mathan, S., Ververs, P.M., Erdogmus, D., Adami, A., Pavel, M., Lan, T.: Supporting Real-time Cognitive State Classification on a Mobile Individual. J. Cog. Eng. \& Decision Making 1(3), 240-270 (2007); Special Issue on Augmented Cognition: Past, Present, and Future

2. Levonian, E.: Retention Over Time in Relation to Arousal During Learning: An Explanation of Discrepant Results. Acta Psychological 36(4), 290-321 (1972)

3. Berka, C., Levendowski, D.J., Lumicao, M.N., Yau, A., Davis, G., Zivkovic, V.T., Olmstead, R.E., Tremoulet, P.D., Craven, P.L.: EEG Correlates of Task Engagement and Mental Workload in Vigilance, Learning, and Memory Tasks. Aviat. Space Environ. Med. 78(5, suppl.), pp. B231-B244 (2007)

4. Klein, G.A.: Seeing the Invisible: Perceptual-Cognitive Aspects of Expertise. In: Rabinowitz, M. (ed.) Cognitive Science Foundations of Instruction, pp. 203-226. Erlbaum, Mahwah (1992)

5. Stevens, R., Galloway, T., Berka, C.: Allocation of time, EEG-engagement and EEG workload resources as scientific problem solving skills are acquired in the classroom. In: Proceedings of 3rd Augmented Cognition International, held in conjunction with HCI International 2007, Beijing, China, July 22-27, Springer, Heidelberg (2007)

6. Poythress, M., Russell, C., Siegel, S., Tremoulet, P.D., Craven, P., Berka, C., Levendowski, D.J.: Correlation Between Expected Workload and EEG Indices of Cognitive Workload and Task Engagement. In: Schmorrow, D., Stanney, K., Reeves, L. (eds.) Augmented Cognition: Past, Present and Future, pp. 32-44. Strategic Analysis, Inc., Arlington (2006)

7. Berka, C., Levendowski, D.J., Cvetinovic, M., Petrovic, M.M., Davis, G.F., Lumicao, M.N.: Real-time Analysis of EEG Indices of Alertness, Cognition and Memory Acquired with a Wireless EEG Headset. Int. J. of Human-Computer Interaction 17(2), 151-170 (2004)

8. Berka, C., Levendowski, D.J., Ramsey, C.K., Davis, G., Lumicao, M.N., Stanney, K., et al.: Evaluation of an EEG-Workload Model in an Aegis Simulation: Biomonitoring for Physiological and Cognitive Performance during Military Operations. Int. Soc. for Opt. Eng. 5797, 90-99 (2005) 
9. Neri, D. F., Dinges, D. F., Rosekind, M.R.: Sustained Carrier Operations: Sleep loss, Performance, and Fatigue Countermeasures. Moffett Field, California: NASA Ames Research Center (1997),

http: / / human-factors.arc.nasa.gov/zteam/PDF_pubs /

Nimitz1997.pdf (accessed July 24, 2009)

10. Schneider, W., Chein, J.M.: Controlled \& Automatic Processing: Behavior, Theory, and Biological Mechanisms. Cog. Sci. 2(7), 525-559 (2003)

11. Kelly, A.M.C., Garavan, H.: Human Functional Neuroimaging of Brain Changes Associated with Practice. Cerebral Cortex 15(8), 1089-1102 (2005)

12. Sonnentag, S.: Excellent Performance: The Role of Communication and Cooperation Processes. App. Psych.: An International Review 49(3), 483-497 (2000)

13. Hoffman, R.R., Feltovich, P.J., Fiore, S.M., Klein, G., Ziebell, D.: Accelerated Learning? IEEE Intelligent Systems 24(2), 18-22 (2009) 